Questions vives

\section{Questions Vives}

Recherches en éducation

$N^{\circ} 28 \mid 2017$

De l'indifférenciation à la différenciation

\title{
Les trois dimensions de la signifiance dans une approche anthropo-philosophique, interlocutive et empirique de la personne en éducation
}

\author{
Marie-Louise Martinez et Tommy Terraz
}

\section{(2) OpenEdition}

Journals

Édition électronique

URL : http://journals.openedition.org/questionsvives/2583

DOI : $10.4000 / q u e s t i o n s v i v e s .2583$

ISSN : $1775-433 \mathrm{X}$

Éditeur

Université Aix-Marseille (AMU)

Édition imprimée

Date de publication : 29 décembre 2017

ISBN : 978-2-912643-52-0

ISSN : $1635-4079$

\section{Référence électronique}

Marie-Louise Martinez et Tommy Terraz, «Les trois dimensions de la signifiance dans une approche anthropo-philosophique, interlocutive et empirique de la personne en éducation », Questions Vives [En ligne], N 28 | 2017, mis en ligne le 15 novembre 2018, consulté le 10 décembre 2020. URL : http:// journals.openedition.org/questionsvives/2583; DOI : https://doi.org/10.4000/questionsvives.2583

Ce document a été généré automatiquement le 10 décembre 2020.

\section{c)}

Questions Vives est mis à disposition selon les termes de la licence Creative Commons Attribution Pas d'Utilisation Commerciale - Pas de Modification 4.0 International. 


\title{
Les trois dimensions de la
} signifiance dans une approche anthropo-philosophique, interlocutive et empirique de la personne en éducation

\author{
Marie-Louise Martinez et Tommy Terraz
}

\section{Introduction}

1 Au regard des confusions actuelles dans la culture et l'éducation entre les domaines disciplinaires (confusions des disciplines souvent engendrées par les nouveaux curricula des éducations à.), l'hybridation anomique des genres du discours, des types de textualités et des domaines symboliques de la pratique (confusion entre le politique et l'économique, entre le religieux et le politique, entre le scientifique et le technologique, etc.), l'enjeu de différencier finement les ordres et les domaines, sans pour autant les disjoindre, devient majeur surtout dans l'éducation et l'enseignement. L'approche interlocutive pragmatique en philosophie de la personne et du texte conçue par Francis Jacques depuis les années 1980 présente alors des outils théoriques et méthodologiques très précieux pour répondre à ces enjeux. En effet, c'est au cœur des pratiques langagières, discursives et textuelles, dans l'espace de l'interlocution (Jacques, 1985), que s'opère non seulement la signification logique, mais aussi l'identification des subjectivités dans l'intersubjectivation et l'émergence des personnes. La confusion anomique, la ségrégation, l'exclusion ou au contraire, l'intégration dans un statut différencié et complémentaire, sont occasionnées dans l'échange langagier, selon la place accordée aux interlocuteurs, particulièrement dans le processus éducatif.

2 L'approche interlocutive de l'émergence de la personne en éducation et en formation, inaugurée depuis 1997 (Martinez, 1997, 2002, 2005, 2010, etc.) demande d'observer 
précisément, à partir des marques langagières, notamment l'énonciation, l'appellation, la pronominalisation et autres indicateurs (Martinez \& Chamboredon, 2011) comment se coconstruisent les identités au sein des interactions langagières et de la relation interlocutive dans la pratique éducative, thérapeutique ou professionnelle (Martinez \& Gadchaux, 2014 ; Martinez \& Terraz, 2016). L'importance est majeure en philosophie de l'éducation de repérer les traces d'une praxéologie de l'émergence de l'élève comme sujet, personne ou citoyen, dans le processus éducatif. La démarche d'analyse interlocutive des textes et des discours, mais aussi des interactions langagières dans la classe, ou d'entretiens, ou encore d'analyse de textes produits avec et par les acteurs éducatifs, permet de voir avec clarté les divers indicateurs dont les échanges ou les textes sont porteurs.

Pour étudier l'échange langagier, en sciences humaines et sociales, c'est sur l'analyse de contenu, sur le lexical simplement, que l'on s'appuie aujourd'hui encore. Or, il est évident qu'une démarche d'analyse s'appuyant sur le repérage des trois dimensions imbriquées de la signifiance, non seulement sémantico-lexicale mais encore syntaxique et pragmatique, peut bien mieux prendre en charge la complexité de la signification et particulièrement les enjeux relationnels des acteurs et de leur identification. Dans l'article, pour situer une démarche originale qui se développe dans la pratique et la recherche en sciences de l'éducation, en anthropologie et philosophie de l'éducation et de la formation, depuis plus de vingt ans maintenant, dans ses références épistémologiques, théoriques et méthodologiques, nous consacrons d'abord un temps de présentation aux notions des trois dimensions de la signifiance, puis nous soulignons l'enjeu de l'intégration du tiers précédemment exclu, enfin nous discutons la possibilité de mettre ces catégories et instances au service d'une démarche empirique en philosophie de l'éducation.

\section{Des trois composantes aux trois dimensions}

4 La trichotomie, sémantique, syntaxique et pragmatique, élaborée par Charles Morris (1964), comme composant la signification à partir de la complexe semiosis du philosophe Charles Sanders Peirce (1839-1914), a fourni des notions opérationnelles et efficaces, exploitées par les linguistes, les sociolinguistes ou les didacticiens de la langue. Cette approche donne lieu à des analyses empiriques de corpus de l'activité de production comme de réception des discours et des textes. Elle a cependant engendré une compréhension un peu réductrice de la théorie de Peirce, que nous ne pourrons pas développer dans le cadre de cet article. Ce philosophe, logicien et épistémologue, qui a le projet de définir une logique des relations (Deledalle, 1990), a élaboré une théorie de la signification qui rend compte des divers domaines culturels (Nicole Everaert-Desmedt, 1990). Il a proposé une réflexion sur le signe antérieure à celle de Saussure mais moins connue en France. Cette théorie sociale et relationnelle fait rupture avec la théorie saussurienne entre autres par la place accordée à une référence externe à la relation entre les acteurs de la signification : « le signe est le signe de quelque chose en dehors de lui, il signifie pour quelqu'un dans une situation sociale et les signes sont en relation d'opposition entre eux » (Deledalle, 1990, p. 117). Cela mériterait d'être davantage étudié, dans la discussion et la complémentarité avec la théorie de Saussure, ce que nous ne pourrons faire dans le cadre de cet article. 
5 C'est à partir d'une relecture de Peirce, dans le souci de rétablir la part de chaque dimension, particulièrement de la référence et de la communicabilité oubliées trop souvent, que Francis Jacques redéfinit les trois composantes de la signification, dans un article intitulé «De la signifiance » publié en 1987 dans la Revue de Métaphysique et de Morale. Jacques souhaite chercher la structure originaire et élémentaire au-delà de la notion de sens commun du signifier : "signifier, c'est utiliser un signe (ou une séquence de signes) pour renvoyer à un sens, et cela à propos d'un monde à dire. Conformément aux suggestions de l'étymologie : signum fieri » (1987, p. 179). À l'instar du projet peircien, il veut décrire les processus complexes de mise en relation entre des interlocuteurs, des objets du monde et des signes, «dorénavant, nous désignerons la totalité du processus par le terme signifiance » (p. 180). Projet ambitieux et opportun pour une démarche en philosophie du langage et plus tard (2007) du texte.

6 Le philosophe décrit ainsi les grandes dimensions de la signifiance dans leur ensemble comme de grandes questions catégorielles permettant de comprendre plus finement la signification textuelle et interlocutive; mais surtout, il en fait trois conditions de possibilité, chacune étant nécessaire mais non suffisante :

[...] mon hypothèse retiendra trois conditions nécessaires :

1) La présence de marques, sons, graphèmes, etc., qui constituent le matériel signifiant d'un système donné de signes. C'est la condition de différence ;

2) La réalité extra-linguistique à quoi se réfèrent les séquences de signes produites en contexte que sont les énoncés. C'est la condition de référence ;

3) Les utilisateurs du langage par lesquels, entre lesquels et pour lesquels les signes signifient quelque chose. C'est la condition d'interlocution. Comme elle est largement inédite, réduite, esquivée, nous la mettrons en relief.

En indiquant que ce sont des conditions nécessaires, nous avançons avec une extrême insistance qu'entre elles il n'y a pas à choisir. Une théorie convenable de la signification devra s'arranger pour les honorer, c'est-à-dire les considérer dans leurs implications respectives, avant de les intégrer toutes les trois.

Différence, référence, interlocution. Trois mots-clefs pour dessiner la structure élémentaire de la signifiance (1987, pp. 182-183).

7 Ces conditions de possibilité de la signification sont placées a priori, pour garantir la qualité optimale de la communication dans la signification. La posture évoquée est celle de Kant, en philosophie transcendantaliste, pour laquelle il existe des catégories qui sont des conditions de possibilité, posées a priori car nécessaires (le temps, l'espace, comme formes a priori de la sensibilité) en amont de l'expérience d'accès à la connaissance pour la raison pure, ou conditions de possibilité de la moralité (impératifs catégoriques, devoirs moraux parfaits) pour la raison pratique. Pourtant, chez Jacques, il s'agit moins de formalisme rationaliste hérité des Lumières, de transcendantalisme de surplomb, que de hardiesse fondationnelle pour arrimer la signification et la communication à la relation, considérée comme originaire. D'où l'expression de primum relationis : c'est la relation qui est première, qui rassemble et différencie les personnes (Jacques, 1982, pp.360-363). C'est la primauté d'un homo interrogans en relation interlocutive qui différencie les textes comme catégories d'interrogation, aux fondements (Jacques, 2007). Ces fondements posent un principe indémontrable, un jugement synthétique a priori, à l'instar de la logique des relations de Bertrand Russel (1872-1970) : précisons que pour Kant, «si toute connaissance débute avec l'expérience, cela ne prouve pas qu'elle dérive toute de l'expérience » (Critique de la raison pure, 1787/1985, Introduction) .

Selon l'option qui fut celle de Russel, on voit que la relation ce n'est plus avoir rapport, c'est être en rapport. Le principe des relations externes veut que la 
relation s'établisse entre deux termes. Elle est comme un troisième entre deux, tiers médiateur, tiers inclus. Elle relate comme un tiers qui est entre l'un et l'autre, bref comme un de plus. Une relation devient aussi réelle que ses termes. Son rôle est de mettre en ordre et de mettre un sens à l'ordre des individus, qui par eux-mêmes sont étrangers à cet ordre. La relation introduit dans l'univers quelque chose d'irréductible aux individus $\mathrm{x}$ et $\mathrm{y}$, qui ne saurait être absorbé par eux : ce quelque chose est un universel (Jacques, 1982, pp. 146-147).

8 Dans le cadre de sa philosophie du texte, vingt ans plus tard, Jacques reprend le qualificatif si important pour la philosophie kantienne et formaliste, qu'il définit de la manière suivante :

Transcendantal: Caractérise une enquête radicale sur les fondations. Nous appelons 'transcendantale' la condition de possibilité de l'homologie de la pensée interrogative à la réalité interrogée. Sur ce plan, l'étude devrait porter sur la critique des modes de l'interroger par rapport à ce qu'ils peuvent réaliser a priori. À ne pas confondre avec la conformité métaphysique de la réalité interrogeante avec la réalité interrogeable (2007, glossaire, p. 341).

9 Les trois dimensions de la signifiance sont aussi, pour Jacques, les conditions de possibilité d'une textualité véritable (2007). L'auteur propose une distinction entre les «textes» ("véridictoires» ou « de légitimation») et les «non-textes, sous-textes» ( "triviaux », « médiatiques", « idéologiques", " pédagogiques», etc.). Cela en considérant qu' " un texte digne de ce nom suppose un travail sur les trois axes de la signifiance - référence, différence, communicabilité - qui ne peut s'accomplir que dans le contexte même de l'œuvre»(2007, p. 270). À l'inverse, les non-textes et sous-textes triviaux « dérogent aux conditions de possibilité de la signifiance du texte » (p. 250), c'està-dire qu'une ou plusieurs des trois dimensions de la signifiance peut être ou est « gravement lésée » (p. 250). Nous devons aussi compter que c'est dans la textualité, mais aussi dans les situations de la vie quotidienne, régulées par l'interlocution, selon les règles des domaines de la pratique et les domaines textuels que se construisent les significations cognitives et textuelles tout autant que l'ordre relationnel et institutionnel, et que les subjectivités elles-mêmes. Ainsi, poser de façon a priori les trois dimensions de la signifiance comme conditions de la textualité pourrait être compris comme étant une tentative d'éviter l'indifférenciation textuelle et de protéger les grands textes face aux risques de confusions des ordres du savoir et de la textualité, aux sous-textes triviaux. Si certains hybrides comme des textes aux caractéristiques croisées et mixtes sont viables et apportent aux carrefours transtextuels de questionnements différents une véritable innovation sémantique (par exemple certains grands textes philosophiques très nourris de poésie ou de religion comme ceux de Lévinas qui ne confondent cependant jamais les ordres textuels), d'autres hybrides restent monstrueux (comme certains textes idéologiques de propagande qui confondent le politique, l'économique et le scientifique).

En outre, il est possible de développer l'usage des trois dimensions de la signifiance, pour étudier l'échange langagier dans le cadre d'une approche empirique interlocutive, pour affronter l'expérience de la communication avec ses transgressions relationnelles ou ses inventions, ses innovations communicationnelles. Il devient possible de repérer et de comprendre les dysfonctionnements, pour mieux les éviter, pour réguler l'agir praxéologique, comme il sied à une philosophie de l'éducation, tournée vers la valeur, transcendante et immanente, de l'émergence de la personne (Martinez, 2002). Cela demande d'avoir statué sur la place du tiers, comme le demande une théorie de la personne non plus moniste (je) ni dyadique (je et tu) mais relationnelle et dialogale qui fait une place structurante et conséquente à l'instance du tiers symbolique mais aussi 
personnel (je tu, il-elle) (Jacques, 1982), transposée en éducation (Martinez, 1997, 2002, 2010).

\section{Le retour de l'absent, l'intégration relationnelle du tiers personnel précédemment exclu}

11 Comme l'épistémologue Michel Serres l'avait analysé en 1966, dans un article trop peu connu intitulé explicitement «Le troisième homme ou le tiers exclu», les conditions logico-anthropologiques de la réussite de la communication, pour une philosophie du langage, prolongeraient en quelque sorte l'impératif de la logique classique, depuis Aristote, reposant sur le principe du tiers exclu c'est-à-dire celui de non-contradiction. Vraie ou fausse est la seule issue pour une proposition, Tertium non datur: le tiers est exclu. Toute vérité doit être tranchée, l'exclusion du flou s'impose dans la recherche cognitive et éthique. Dans le dialogue, il s'agirait moins d'un combat ou débat entre deux antagonistes que de l'éviction du tiers comme bruit, le troisième homme qui brouille la communication, l'exclu :

[...] une sorte de jeu pratiqué par les deux interlocuteurs, considérés comme associés, contre les phénomènes de brouillage et de confusion, voire contre des individus ayant quelque intérêt à rompre la communication. [...] on les considère comme disputant de conserve contre un ennemi commun [...] Ils luttent ensemble pour l'émergence d'une vérité [...] contre le brouillage, contre le démon, contre le Troisième homme (Serres, 1966, p. 465).

C'est toujours l'éviction du troisième homme qui scellerait le pacte communicationnel, deux contre un, comme l'avait déjà bien montré Benveniste (1974). « Dès que le pronom je apparaît dans un énoncé où il évoque - explicitement ou non - le pronom tu pour s'opposer ensemble à il, une expérience humaine s'instaure à neuf et dévoile l'instrument linguistique qui la fonde » (Benveniste, 1974, p. 68.). Le tiers délocuté, selon l'expression de Benveniste, celui dont on parle, fonde l'interlocution habituelle tout en en faisant les frais, le linguiste décrit le statut très particulier, fragile et ambivalent, du tiers personnel et de la troisième personne :

La conséquence doit être formulée nettement: la troisième personne n'est pas une personne; c'est même la forme verbale qui a la fonction d'exprimer la nonpersonne. [...] De sa fonction de forme non personnelle, la troisième personne tire cette aptitude à devenir aussi bien une forme de respect qui fait d'un être bien plus qu'une personne qu'une forme d'outrage qui peut le néantiser en tant que personne (Benveniste, p. 228-231).

Le linguiste Benveniste dévoile sans ambiguité, comme fait, une structure d'éviction fondatrice de toute expérience linguistique, celle de l'exclu, qui fonde le pouvoir du symbolique. Mais comme le souligne Michel Serres, à travers l'éviction du troisième homme, c'est aussi, sur le plan de la quête scientifique, dans l'approche épistémologique, l'empirique qui est visé par le formalisme rationaliste :

Formaliser, en général, c'est accomplir un processus par lequel on passe de modes de penser concrets à une ou des formes abstraites ; c'est également éliminer le bruit de manière optimale. [...] j'élimine l'empirique, je dématérialise le raisonnement. [... ] Exclure l'empirique c'est exclure la différenciation, la pluralité des autres que recouvre le même. [...] Le premier troisième homme à exclure c'est l'empiriste, le premier tiers à exclure c'est l'empirie... (Serres, 1966, pp. 467-468). 

l'intégration du tiers, comme valeur, la fois cognitive, éthique et praxéologique. Francis Jacques, dans son ouvrage Différence et subjectivité publié en 1982, inversait la fatalité du deux-contre-un, il analysait le dialogue comme articulation relationnelle qui ne produit de signification que grâce à l'intégration du troisième homme précédemment exclu. La personne, comme sujet en relation émerge sous condition interlocutive tri-actancielle. Pas de subjectivation, encore moins de construction personnelle, pas d'innovation sémantique, ni même de signification, sans faire une place au tiers: "Il n'y a pas d'identité personnelle sans un certain rapport au tiers absent ou lointain. On devra prendre en compte, pour l'intégrer, la troisième personne " (Jacques, 1982, p. 52).

Dans cette philosophie pragmatique et interlocutive de la personne, l'exigence éthique et cognitive à la fois demande d'affronter la réalité de l'éviction en vue de permettre «le retour de l'absent et de l'exclu ». Pourquoi alors entériner l'éviction de l'empirique et le troisième homme dans une posture épistémologique formaliste ? Si l'on prend en compte l'argument de Michel Serres consistant à pointer du doigt une certaine exclusion de l'empirique par le formalisme rationaliste, et que nous l'associons à l'exigence jacquéenne de l'intégration du tiers, non seulement comme relation ou comme tiers code mais aussi comme tiers personnel, alors il semble légitime de penser que l'investigation empirique des échanges langagiers revêt une grande importance. Il semble possible et nécessaire, dès lors, de faire place à une pragmatique personnaliste empirique dans ses démarches praxéologiques et méthodologiques en philosophie de l'éducation. L'article, après la réflexion épistémologique et théorique, aborde les règles méthodologiques de la démarche, se contentant d'évoquer, compte tenu du manque de place, quelques exemples d'analyse de corpus en anthropologie philosophique pour l'émergence de la personne en l'éducation.

\section{Pour une pragmatique personnaliste interlocutive empirique}

16 La philosophie de l'éducation dans le cadre des sciences de l'éducation a entre autres rôles à dessiner les démarches praxéologiques et méthodologiques d'une pragmatique personnaliste pour l'éducation mais aussi pour la formation et la professionnalisation. Loin de se cantonner à une approche aprioriste et formaliste, elle peut selon une approche empirique, prendre en compte la présence du tiers dans sa singularité concrète, dans l'échange langagier dans la classe ou ailleurs. Cela est très important, entre autres, dans la professionnalisation pour définir les pratiques optimales en pédagogie, didactique, éducation et formation. Cette approche empirique permet dans la praxis éducative-formative, comme dans la recherche, d'objectiver sans les réifier les situations éducatives pour mieux les critiquer, les réguler, les transformer.

Quels seraient alors les protocoles méthodologiques de l'analyse interlocutive de corpus?

L'intérêt de la démarche en philosophie et sciences de l'éducation est de réguler l'agir praxéologique en éducation et de développer une analyse de discours cohérente sur les plans méthodologique, théorique et épistémologique. Les analyses issues de ces protocoles de recueil, de transcription et d'analyse des données interlocutives permettent aux acteurs et aux chercheurs de mieux voir, comprendre, interpréter la "réalité » des interactions langagières ou des entretiens. Tentons à présent de détailler plus 
précisément les paramètres de l'analyse interlocutive selon les trois dimensions de la signifiance.

18 1) La dimension de la Différence correspond aux questions catégorielles - comment? Au moyen de quels matériaux langagiers? - elle renvoie au rapport des signes avec les autres signes. La dimension de la différence est donc une dimension morpho-syntaxique et linguistique, sur l'axe sémiotique. Est étudiée la structure dynamique du texte, sa construction, son mouvement, pour repérer et dégager le plan, les connecteurs logiques, les adverbes de liaison, etc. On peut y reconnaître les types discursifs (descriptif, narratif, explicatif, argumentatif, incitatif, prescriptif, etc.) dans leur syntaxe particulière, leur fonction pour le locuteur. À quelle(s) catégorie(s) et type(s) le texte appartient-il ? (scientifique, philosophique, littéraire, journalistique, religieux, etc.) À quels (s) genre(s) textuels ? Le sujet est-il dans la description, la narration, l'injonction?

2) La dimension de la Référence correspond à la question catégorielle - de quoi parle-ton? Ici, le sens renvoie au rapport des signes avec les signifiés mais aussi les objets de référence, à l'en-dehors du langage, aux thématiques, aux objets, notions, concepts. La dimension de la Référence est donc une dimension sémantique. Une analyse lexicale, thématique et conceptuelle devient possible, consistant à dégager les thèmes, les notions, les concepts principaux, les grandes catégories, etc.

20 3) La dimension interlocutive, pragmatique, relationnelle : la Communicabilité correspond aux questions catégorielles - qui parle à qui ? - et - pourquoi ? - (pour quelle(s) raison(s), cause(s) ?) - pour quoi? - (dans quel(s) but(s) ?) Quelle interlocution? Dialogisme interne/externe? Quelle implication de l'auteur(e) ? Quels marqueurs énonciatifs, interlocutifs? Ici le sens renvoie aux rapports des signes avec les usagers des signes et avec les enjeux de l'échange langagier. On agit avec des mots ou des actes de langage, pas d'énoncé sans énonciation, pas d'énonciation sans interlocution: tout énoncé est acte langagier, situé dans un échange, dialogique, fût-il imaginaire ou intériorisé : 'No private langage', comme soulignait Wittgenstein. Quels inter-actes de langage du (de la) locuteur (e) dans l'interlocution avec son (sa, ses) interlocuteur(e.s) ? La communicabilité signale les enjeux relationnels, les problèmes, la tension dialogique, entre les interlocuteurs.

21 Dès lors, il devient possible de voir et repérer la co-construction de significations et d'identités dans les échanges langagiers. Cela n'est pas anodin pour penser l'éducation dans la mesure où l'on retrouve les trois conditions de possibilité ou finalités de toute éducation ou formation dans l'échange langagier :

a. la co-construction de significations cognitives, discursives, textuelles et leur transmissionappropriation ;

b. la co-construction d'identités sociales, cognitives, épistémiques, citoyennes, morales, etc.;

c. la co-construction d'identités subjectives d'individu (sortir des mimétismes subis vers un processus d'individuation différencié); de sujet (intégration des codes symboliques et des interdits de l'ordre symbolique); de personne (intégration du tiers précédemment exclu et d'une différenciation personnelle tri-instancielle); de citoyen (rapport avec l'autre dans la cité commune); d'éco-citoyen (quelle place faite à l'autre environnement, l'autre espèce dans un monde partagé ?)

En fonction des diverses hypothèses, le chercheur sélectionne les indicateurs à rechercher. Il rassemble des indices, procède en ethnographe en réunissant les marques pour établir des preuves. Il procède en ethnologue en les mettant en forme (dégager des processus sous-jacents), pour repérer les pistes d'interprétation. Il procède en 
anthropologue en permettant une universalisation de ce modèle d'intelligibilité. Objectivation empirique jamais réifiante, le chercheur, en philosophe, repère dans l'interagir humain les finalités (différenciation épistémique et personnelle, différenciation des textes) à l'œuvre pour réguler la praxéologie éducative.

Ici, on peut chercher les manifestations de l'interpellation à l'adresse des interlocuteurs, selon la qualité de la relation interlocutive, l'interpellation, entre autres actes langagiers, peut en effet contribuer à assujettir ou instituer les sujets (Martinez, 2010), jusqu'à la différenciation par le recouvrement interlocutif de trois instances de la personne (Jacques, 1982, p. 51). Dans un rapport de «sui-référenciation" et de "rétroréférenciation" (Jacques, 1985), les acteurs peuvent évoquer leur propre construction identitaire, en se désignant eux-mêmes, dans le passé ou le futur, ou même dans le présent de manière réflexive. Ils peuvent le faire grâce à l'écoute éducative, thérapeutique, accompagnatrice de l'autre, dans le rapport à l'altérité non seulement dialogique mais dialogale qui sait toujours faire place au tiers. Rapport de subjectivation et d'intersubjectivation dans l'émergence de la personne qui fait place aux trois instances personnelles (je-tu-il/elle) dans l'interlocution. Les identités se définissent interlocutivement dans l'interagir humain. Le sujet parlant, contraint et agissant, se définit lui-même dans son identité dans des situations d'interlocution où la réflexivité compose avec l'altérité.

On peut objectiver les interactions entre les acteurs, les rendre visibles, sans jamais les réifier, à travers les indicateurs langagiers, analyser avec les outils de l'analyse linguistique et pragmatique, dans leurs processus d'intersubjectivation et d'interpersonnification. Un véritable savoir sur l'homme en éducation et en formation devient possible, on peut observer, objectiver l'autre et même "soi-même comme un autre » (Ricœur, 1990), sans cesser pour autant d'être sujet. Il devient très facile dès lors de repérer les manifestations de violence qui guettent toujours les situations éducatives. Il devient possible de réguler l'indifférenciation comme la différenciation ségrégative des savoirs et des personnes pour s'engager dans un agir éducatif de l'émergence de la personne, par et pour la compréhension des pratiques textuelles des cultures. On peut dès lors repérer les processus d'émergence de la personne (Martinez, 2002), quand le recouvrement empirique, a posteriori des trois instances interlocutives, vient répondre aux conditions définies a priori. La mise en pratique de cette démarche interlocutive personnaliste empirique, en philosophie de l'éducation, appuyée sur les trois dimensions de la signifiance, a déjà donné lieu à la publication de plusieurs travaux. Dans certaines recherches il a été possible de montrer l'indifférenciation violente dans la classe, les moments de chaos anomiques, avec une interpellation indifférenciatrice qui amène très vite les élèves à une collusion contre certains élèves ou contre le savoir ou l'enseignant(e) qui vont être les exclus de la communication (Martinez, 2010). Il est possible de voir comment et pourquoi l'usage interlocutif de l'interpellation en classe peut contribuer à l'indifférenciation chaotique des élèves et des savoirs dans une classe de collège qui se résout sur deux processus d'éviction du tiers: un élève bouc émissaire comme tiers personnel, et le savoir, du texte pédagogique historique comme tiers symbolique. Dans une autre classe de collège, au contraire, la démarche praxéologique permet un double processus de différenciation des élèves comme sujets et personnes avec la différenciation des savoirs langagiers et disciplinaires. Il a été possible dans une formation à la citoyenneté avec des adultes de montrer dans la praxéologie éducative du débat, le passage d'interactions chaotiques avec production violente du bouc émissaire vers une 
socialisation et personnalisation écocitoyenne (Martinez \& Chamboredon, 2011). Nous avons, enfin, pu étudier la communication de partenaires avec des dysfonctionnements délocutifs, des effets d'éviction ou au contraire des moments réparateurs d'intégration des membres les plus fragiles dans la communication éducative ou thérapeutique (Martinez \& Gadchaux, 2014 ; Martinez \& Terraz, 2016).

\section{Conclusion}

L'approche, ici présentée, peut être qualifiée d'anthropo-philosophique interlocutive et empirique de la personne en éducation. Construite autour des trois dimensions de la signifiance de Francis Jacques, qu'elle s'est efforcée de transposer en philosophie et sciences de l'éducation, elle a pu montrer l'importance de maintenir certaines conditions de possibilité de nature philosophique. On ne saurait, en effet, se passer des conditions de possibilité que sont les grandes finalités de l'éducation comme l'émergence de l'élève au statut relationnel de sujet, personne ou citoyen pour penser philosophiquement l'éducation. De même on ne saurait se passer des trois dimensions de la signifiance pour analyser les conditions de possibilité de la textualité dans leur généralité et dans leurs différenciations spécifiques, comme textes de la culture et des savoirs. Pour autant, si les idéaux $\mathrm{du}$ formalisme rationaliste, spéculatifs et abstraits, des philosophies transcendantales ont l'immense mérite de dire les fondements et les finalités, ils n'ont pas pour autant vocation à occulter les fondations anthropologiques violentes quand elles excluent le tiers.

L'investigation sur la réalité des échanges langagiers interlocutifs, dans l'espace de la relation interlocutive, dans la famille, à l'école, au travail, dans ses fonctionnements et dysfonctionnements, demande de scruter attentivement ce qui fait obstacles, entraves et difficultés ou au contraire ce qui favorise l'accès à la relation dialogale véritable, triinstancielle. L'analyse empirique des marques et des indicateurs langagiers est indispensable pour rendre visibles les processus de l'éviction du tiers et de son intégration possible. Tendre vers une démarche pragmatique personnaliste empirique, en philosophie de l'éducation, appuyée sur les trois dimensions de la signifiance de Francis Jacques, c'est engager une double approche épistémologique, dans l'articulation cohérente. C'est maintenir une approche philosophique, à partir de conditions de possibilités posées a priori comme repères fondateurs de la textualité, du dialogue, de l'éducation, autorisant l'émergence des savoirs et des personnes, tout en se donnant les moyens de mettre en œuvre une approche empirique. Dans la démarche épistémologique et méthodologique de cette analyse interlocutive de la communication langagière, on devrait pouvoir se dégager tout à la fois des impasses idéalistes du formalisme philosophique et des impasses positivistes qui nient et occultent la subjectivité. La question vive ici porte sur la contribution à l'hypothèse audacieuse et controversée de la possibilité d'une approche empirique en philosophie. Nous la fondons ici sur la priorité de l'exigence éducative. 


\section{BIBLIOGRAPHIE}

Benveniste, E. (1974). Problèmes de linguistique générale Tome II : La communication. Paris : Gallimard.

Everaert-Desmedt, N. (1990). Le processus interprétatif, introduction à la sémiotique de Ch. S. Peirce.

Liège : Mardaga.

Deledalle, G. (1990). Lire Peirce aujourd'hui. Bruxelles : De Boeck Université.

Jacques, F. (1982). Différence et subjectivité. Anthropologie d'un point de vue relationnel. Paris : Aubier Montaigne.

Jacques, F. (1985). L'espace logique de l'interlocution. Paris : PUF.

Jacques, F. (1987). De la signifiance. Revue de métaphysique et de morale, 2, (« Philosophie du langage »), avril-juin, 179-218.

Jacques, F. (2000). Écrits anthropologiques. Paris : L'Harmattan.

Jacques, F. (2007). L'arbre du texte et ses possibles. Problèmes et controverses. Paris : Vrin.

Kant, E. (1985). Critique de la raison pure (1787). Paris : Gallimard.

Martinez, M.-L. (1997). Vers la réduction de la violence à l'école ; contribution à l'étude de quelques concepts pour une anthropologie relationnelle de la personne. (Thèse de doctorat sous la direction de Francis Jacques). Lille : Septentrion.

Martinez, M.-L. (dir.) (2002). L'émergence de la personne. Éduquer, accompagner. Paris : L'Harmattan.

Martinez, M-L. (2005). Le débat comme espace interlocutif d'identification des textes et des personnes. Tréma (IUFM de Montpellier), 24, Dossier « approche anthropologique en éducation et en formation » Tome 2, Coord. M.-L. Martinez, 77-101.

Martinez, M.-L. (2010). L'interpellation en classe, entre indifférenciation et différenciation des personnes. Corela (Cognition, Représentation, Langages) du Cercle 10 Linguistique du Centre et de l'Ouest. Repéré à http://corela.revues.org/1033

Martinez, M.-L. \& Chamboredon, M-C. (2011). Approche anthropologique de la co-construction d'identités citoyennes ; l'exemple de discussions sur le développement durable, dans l'enseignement professionnel. Dans L. Simonneaux et A. Legardez (dir) Questions socialement vives et durabilité (pp. 89-113). Dijon : Educagri-éditions.

Martinez, M.-L. \& Gadchaux, C. (2014). L'appui des approches relationnelles pour favoriser l'accessibilité à l'université. La nouvelle revue de l'adaptation et de la scolarisation, 67, 193-208. Repéré à http://www.cairn.info/revue-la-nouvelle-revue-de-l-adaptation-et-de-la-scolarisation-2014-3page-193.htm

Martinez, M.-L. \& Terraz, T. (2016). Partenariat et coordination pluriprofessionnelle - Approche relationnelle et interlocutive de la personne. Carrefours de l'éducation, 42, 61-74. Repéré à http:// www.cairn.info/revue-carrefours-de-l-education-2016-2-page-61.htm. DOI : 10.3917/ cdle.042.0061

Morris, C.W. (1964) Signification and Signifiance, Cambridge (Mass.) : MIT Press.

Ricœur, P. (1986). Du texte à l'action, Essais d'herméneutique, II. Paris : Seuil. 
Ricœur, P. (1990). Soi-même comme un autre. Paris : Seuil.

Serres, M. (1966). Le troisième homme ou le tiers exclu. Les études philosophiques, 4, octobredécembre, 463-470.

\section{RÉSUMÉS}

Les trois composantes de la signification décrites par Morris (1964) à partir de Peirce, reprises en linguistique et didactique sont redéfinies en philosophie par Francis Jacques (1987) comme les trois dimensions - référence, différence, communicabilité - érigées de façon aprioriste au statut de conditions de possibilité de la signifiance et de la textualité. Dans l'article, après avoir présenté les enjeux de cette catégorisation des trois dimensions qui permet de différencier des textes, nous évoquons la transposition réalisée à partir de l'approche épistémologique a priori jacquéenne vers une approche empirique. Une telle démarche, à la croisée de la philosophie de l'éducation et de la philosophie pragmatique et interlocutive du langage et de la personne, audelà de l'observation de la différence des textes permet de repérer la co-construction des personnes dans l'échange interlocutif. Le passage est justifié dans son opportunité grâce à la question de l'intégration du tiers précédemment exclu que la pragmatique transcendantale de Jacques $(1982,1985)$ permet de poser mais non de résoudre. Sur le plan épistémologique, l'approche empirique manifeste la présence du tiers que le formalisme transcendantal évince, comme le voit Michel Serres (1966). L'apport théorique tant pour les professionnels de l'éducation que pour les chercheurs, qui peuvent observer sans réifier les personnes, est considérable. Sur le plan méthodologique on peut objectiver l'émergence de la personne dans les marques langagières de la relation interlocutive éducative et professionnelle.

The three components of meaning described by Morris (1964) from Peirce, used in linguistics and didactics, are redefined in philosophy by Francis Jacques (1987) as the three dimensions = reference, difference, communicability set up aprioristically to the status conditions of possibility of signifiance and textuality. In the article, after having presented the issues of the categorization of the three dimensions that makes it possible to differentiate texts, we evoke the transposition carried out from the a priori Jacquerian epistemological approach towards an empirical approach. Such an approach, at the crossroads of the philosophy of education and the pragmatic and interlocutory philosophy of language and person, beyond the observation of the difference of the texts makes it possible to identify the co-construction of personal identities in the interlocutory exchange. The passage is justified in its opportunity thanks to the question of the integration of the formerly excluded third that the transcendental pragmatics of Jacques $(1982,1985)$ allows to ask but not to solve. On the epistemological level, the empirical approach manifests the presence of the third which transcendental formalism crowds out, as Michel Serres (1966) sees. The theoretical contribution for both education professionals and researchers, who can observe without reifying people, is considerable. On the methodological level we can objectify the emergence of the person in the linguistic marks of the educational and professional interlocutive relationship.

\section{INDEX}

Mots-clés : approche interlocutive, trois dimensions de la signifiance, praxéologie de la personne, tiers exclu et inclus, démarche empirique

Keywords : interlocutive approach, three dimensions of signifiance, praxeology of the person, excluded and included third, empirical approach 


\section{AUTEURS}

\section{MARIE-LOUISE MARTINEZ}

Professeure des Universités, Laboratoire CIVIIC, Université de Rouen

\section{TOMMY TERRAZ}

Doctorant ( $4^{\mathrm{e}}$ année), Laboratoire CIVIIC, Axe « Valeurs et Idées », Université de Rouen, Membre associé au Laboratoire LISEC, équipe « Normes et Valeurs », Membre de la SOFPHIED (Société francophone de philosophie de l'éducation) 